490 women underwent the procedure during the follow-up period. From the observed cancer incidence rate, the risk of ovarian cancer up to age 75 years was estimated at $62 \%$ for $B R C A 1$ mutation carriers, and at $18 \%$ for $B R C A 2$ mutation carriers. The researchers suggest a possible link between previous breast cancer history and development of ovarian cancer. The risk of peritoneal cancer in the 20 years following oophorectomy was $4.3 \%$-a greater incidence than for noncarriers - although the authors suggest that these cancers might have arisen from metastases of subclinical disease present at the time of surgery.

The results of the study support the use of prophylactic salpingo-oophorectomy as protection against ovarian cancer in women carrying BRCA mutations. The authors recommend that both ovaries and fallopian tubes should be removed as potential sites of cancer origin, and that a detailed examination of the removed organs is required to eliminate the possibility of microscopic disease.

Original article Finch A et al. (2006) Salpingo-oophorectomy and the risk of ovarian, fallopian tube, and peritoneal cancers in women with a BRCA1 or BRCA2 mutation. JAMA 296: 185-192

\section{Circulating tumor cell levels can predict survival in metastatic breast cancer}

Methods currently used to assess clinical benefit of systemic treatment for metastatic breast cancer (MBC), such as physical examinations, radiological studies and serologic testing, are often inaccurate early in the disease course. Inaccurate assessment can lead to prolongation of treatment with an inactive therapy, or premature discontinuation of a potentially useful therapy. A previous study demonstrated that elevated levels of circulating tumor cells (CTCs) before initiation of a new therapy or 3-5 weeks later are associated with rapid disease progression and death in patients with MBC. Additional follow-up data have now been reported.

The study enrolled 177 patients with MBC who were about to start a new systemic therapy. CTC levels were recorded before initiation of therapy (baseline), and 3-5, 6-8, 9-14 and 15-20 weeks after therapy was started. Survival analyses were performed using a threshold elevated CTC level of $\geq 5$ CTCs $/ 7.5 \mathrm{ml}$ blood.
At each blood draw, patients with $<5$ CTCs/ $7.5 \mathrm{ml}$ blood showed significantly longer median progression-free survival times than patients with $\geq 5 \mathrm{CTCs} / 7.5 \mathrm{ml}(\geq 6.0$ months vs $\leq 3.6$ months at each blood draw; $P<0.05$ for all comparisons). Median overall survival was also significantly longer in patients with CTC counts below the threshold level $(\geq 18.6$ months vs $\leq 10.9$ months at each blood draw; $P \leq 0.001$ for all comparisons).

The authors conclude that detection of elevated CTC levels at any point during therapy can be used to predict disease progression and mortality in patients with $\mathrm{MBC}$, and might be useful in determining when an alternative therapy is required.

Original article Hayes DF et al. (2006) Circulating tumor cells at each follow-up time point during therapy of metastatic breast cancer patients predict progression-free and overall survival. Clin Cancer Res 12: 4218-4224

\section{PET is predictive of outcome in patients with Hodgkin's disease}

In the treatment of Hodgkin's disease, early identification of patients unresponsive to standard treatment would allow intensification of their therapy. Researchers in Italy have assessed the predictive value of ${ }^{14} \mathrm{~F}$-fluorodeoxyglucose PET in determining the response to chemotherapy, in a prospective study of 40 patients with Hodgkin's disease.

Patients were assessed by PET after 2 cycles of chemotherapy; 28 patients were classified as PET-negative, 8 patients were PETpositive, and 4 patients had minimal residual uptake (MRU). At the end of six cycles of treatment, seven of the eight PET-positive patients remained positive for disease, with the remaining patient classified as having MRU; however, this patient relapsed 3 months after the end of treatment. All other patients were PET-negative, although one patient previously classified as having MRU later suffered a relapse. None of the 28 patients initially classified as PET-negative relapsed within 1 year of treatment, whereas all PETpositive patients had either relapse or disease progression. CT and PET were used for clinical re-evaluation after four and six cycles of therapy. PET was more accurate than CT for the evaluation of early response; only one patient was found to be positive by CT after 